\title{
Risk factors associated with aortic remodeling in patients with Stanford type B aortic dissection after thoracic endovascular aortic repair
}

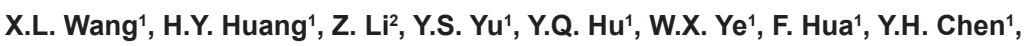
H. Ni', Q.W. Ding ${ }^{1}$ and Z.Y. Shen ${ }^{1}$

${ }^{1}$ Department of Cardiovascular Surgery, First Affiliated Hospital of Soochow University, Suzhou, Jiangsu Province, China

2Department of Interventional Radiology, First Affiliated Hospital of Soochow University,

Suzhou, Jiangsu Province, China

Corresponding author: Z.Y. Shen

E-mail: zhenya_shen@sina.cn

Genet. Mol. Res. 14 (4): 11692-11699 (2015)

Received February 28, 2015

Accepted August 20, 2015

Published October 2, 2015

DOI http://dx.doi.org/10.4238/2015.October.2.2

ABSTRACT. To determine the risk factors associated with adverse aortic remodeling after thoracic endovascular aortic repair (TEVAR) in patients with Stanford type B aortic dissection, we performed a retrospective analysis of 54 patients between January 2009 and June 2012 at the First Affiliated Hospital of Soochow University. All patients underwent TEVAR of the descending thoracic aorta. Multiple-logistic regression analyses were performed to identify risk factors associated with aortic remodeling. Truelumen and false-lumen volumes were increased $(P<0.001)$ and decreased $(P<0.001)$ after surgery, respectively. Therefore, the remodeling index increased after surgery $(1.04 \pm 0.6$ to $2.06 \pm 1.12, \mathrm{P}<0.001)$. Remodeling index and true-lumen volume were higher in the favorable aortic remodeling group compared to the adverse aortic remodeling group $(P<0.001)$, while the false-lumen volume was lower in the favorable aortic remodeling group 
$(P<0.001)$. Multivariate analyses revealed a branch originating from the false lumen $(O R=39.9, P<0.01)$ and multiple tears $(O R=27.4, P<0.01)$ to be independent risk factors for adverse aortic remodeling. Therefore, a branch originating from the false lumen and multiple tears were determined to be independent risk factors for adverse aortic remodeling after TEVAR in patients with Stanford type B aortic dissection.

Key words: Aortic dissection; Aortic remodeling; Lumen volume; Thoracic endovascular aortic repair; Remodeling index

\section{INTRODUCTION}

Aortic dissection is caused by a tear in the aortic intima, which allows the blood to force the intima and the media apart, thus creating a false lumen (Nienaber and Eagle, 2003). The propagation of the tear is responsible for the clinical manifestations of aortic dissection, including pain, hemorrhage, hypertension, and ischemia (Nienaber and Eagle, 2003). The incidence of aortic dissection is between 2.6 and 3.5 per 100,000 person-years, and is known to increase every year (Bickerstaff et al., 1982; Meszaros and Morocz, 2000). Based on the affected part of the aorta, aortic dissections can be classified into ascending (Stanford type A) or descending (Stanford type B) types (Daily et al., 1970). Almost all type A aortic dissections are emergencies that must be treated via immediate surgery, while most type B dissections can be treated medically (only approximately $20 \%$ require surgery) (Erbel et al., 2001; Fattori et al., 2008). Patients with type B dissections requiring surgery may be subjected to open surgery or thoracic endovascular aortic repair (TEVAR) (Dake et al., 1999). Studies have shown that in-hospital mortality rates are much higher for open surgery (around 40\%) than for TEVAR (around 10\%) (Dake et al., 1999; Trimarchi et al., 2006; Fattori et al., 2008; Rodriguez et al., 2008), while a long-term follow-up study showed that the open surgery and TEVAR approaches had similar long-term survival rates (Eid-Lidt et al., 2013). The desired outcomes of surgical repair of type B dissection are an enlarged true lumen and a decreased false lumen, which are associated with favorable aortic remodeling and a good prognosis (Huptas et al., 2009).

Studies showed that a favorable aortic remodeling was not common, with the factors predicting favorable remodeling remaining obscure (Miller and Pierson, 2011; Yang et al., 2012). Therefore, the present study aimed to investigate the independent factors involved in aortic remodeling after TEVAR. A better knowledge of these risk factors might help identify patients at higher risk of treatment failure and consequently perform a closer follow-up in such cases.

\section{MATERIAL AND METHODS}

\section{Patients}

This retrospective study included patients diagnosed with Stanford type B aortic dissection between January 2009 and June 2012, and who were treated with TEVAR at the First Affiliated Hospital of Soochow University. Inclusion criteria were as follows: 1) presence of abdominal aortic aneurysm; 2) presence of a preoperative false lumen without thrombus; and 3) complete computed tomography angiography (CTA) data, including data obtained during diagnosis, and for at least 6 months of postoperative follow-up. Exclusion criteria were as follows: 1) presence of Marfan 
syndrome; 2) descending aortic intramural hematoma; or 3) traumatic dissection. A total of 54 patients met the criteria (40 males and 14 females). The protocol was approved by the Ethics Committee of the First Affiliated Hospital of Soochow University, and written informed consent was obtained from each participant.

\section{TEVAR procedure}

The indications for TEVAR in the selected Stanford type B aortic dissection patients were as follows (multiple indications were possible within a single patient): aortic dissection rupture (20 cases with pleural effusion), chronic chest pain (30 cases), visceral ischemic symptoms (5 cases), and rapid aortic expansion (10 cases). TEVAR was performed according to the technique described by Dake et al. (1999). When the dissection rupture was located near the left subclavian artery $(<1.5 \mathrm{~cm})$, there was a possibility that the stent-graft may cover the left subclavian artery origin. Vertebral artery bypass graft was performed based on the preoperative aortic angiography findings of the ring of Willis and the development of vertebral artery.

\section{Follow-up}

In this study, all patients were preoperatively diagnosed using a large blood vessel coronary computed tomography angiogram (CTA). All patients were subjected to follow-ups after discharge with CTA examinations, for at least 6 months. The CTA scan ranged from the aortic arch to the bilateral femoral artery, using a Somatom Definition FLASH CT System (Siemens, Erlangen, Germany) with the following settings: $128 \times 0.6 \mathrm{~mm}$ collimation, pitch $=1.2$, and $120 \mathrm{kV}$ tube current, using Care4D. Scanning was initiated $8 \mathrm{~s}$ after intravenous administration of $80 \mathrm{~mL}$ Ultravist nonionic contrast material (Schering, Berlin, Germany) at a concentration of $350 \mathrm{mg}$ iodine $/ \mathrm{mL}$, and a flow rate of $5 \mathrm{~mL} / \mathrm{s}$. The assessed indicators included the number of preoperative tears, the preoperative presence of a branch originating from the false-lumen volume, the preoperative true-lumen volume, the preoperative false-lumen volume, the postoperative true-lumen volume, and the postoperative false-lumen volume. The postoperative indicators were based on the final follow-up data obtained. CT image data were computed using a built-in workstation and the Volume software (Siemens), based on the methods described by Wheatley et al. (1995), Singh-Ranger et al. (2000), and Czermak et al. (2004). Because of the individual differences, the absolute value of true- and false-lumen volumes does not adequately reflect the postoperative aortic remodeling outcome (Huptas et al., 2009). Therefore, we calculated the ratio between true- and false-lumen volumes, which is the remodeling index (RI) (Huptas et al., 2009).

\section{Statistical analysis}

All statistical analyses were performed using SPSS 19.0 (SPSS Inc., Chicago, IL, USA). Continuous variables are reported as means \pm standard deviations (SD), and were compared using the Student $t$-test. Categorical variables are reported as proportions, and were analyzed using the $\chi^{2}$ test. Binomial logistic regression analysis was used to determine the independent factors associated with aortic remodeling. Variables with a $P$ value $<0.2$ in univariate analyses were included in the model. In multivariate analyses, a $P$ value $<0.01$ was considered to be significant. 


\section{RESULTS}

\section{Patient and surgical characteristics}

This study included 54 patients, 40 males and 14 females, aged $54.4 \pm 12.5$. Thirty-nine patients had concomitant hypertension, 37 patients had a history of smoking, 18 patients had diabetes mellitus, 11 patients had a peripheral vascular disease, 10 patients had a cerebrovascular disease, and 3 patients had renal dysfunction. Among the 54 patients, 31 were treated using a Talent stent (Medtronic, Fridley, MN, USA), and 23 were treated using an Aegis stent (Microport Medical, Shanghai, China). All dissections were successfully blocked at the proximal side of the tear, and no endoleaks were visualized using CTA. None of the patients died within the first postoperative month. The dissection expanded to the ascending aorta in one patient; this patient recovered well after a second surgery. One patient underwent left-to-right subclavian artery bypass grafting because of left vertebral artery agenesis. Another patient underwent left carotid artery-toleft anterior artery bypass grafting, because the tear was located $1 \mathrm{~cm}$ distal to the orifice of the left subclavian artery.

\section{Remodeling index after TEVAR}

The preoperative and postoperative true-lumen volume, false-lumen volume, and RI are listed in Table 1. The true-lumen and false-lumen volumes were significantly increased after surgery (from $151.76 \pm 36.61$ to $191.89 \pm 39.77 \mathrm{~mL}, \mathrm{P}<0.001$ ) and decreased (from $213.54 \pm 46.64$ to $168.15 \pm 49.70 \mathrm{~mL}, \mathrm{P}<0.001)$ after surgery, respectively. Therefore, there was a significant increase in the postoperative RI compared to the preoperative RI $(1.29 \pm 0.60$ vs $0.77 \pm 0.34, \mathrm{P}<$ $0.001)$. These results indicate that TEVAR can promote aortic remodeling.

Table 1. True lumen volume, false lumen volume, and RI before and after TEVAR in patients with Stanford type B aortic dissection.

\begin{tabular}{lccr}
\hline & Pre-TEVAR & Post-TEVAR & P value \\
\hline True-lumen volume $(\mathrm{mL})$ & $151.76 \pm 36.61$ & $191.89 \pm 39.77$ & $<0.001$ \\
False-lumen volume $(\mathrm{mL})$ & $213.54 \pm 46.64$ & $168.15 \pm 49.70$ & $<0.001$ \\
RI & $0.77 \pm 0.34$ & $1.29 \pm 0.60$ & $<0.001$ \\
\hline
\end{tabular}

TEVAR: thoracic endovascular aortic repair; RI: remodeling index.

\section{Comparison between favorable and adverse aortic remodeling after TEVAR}

Patients were divided into two groups according to the presence of complete thrombosis in the false lumen after TEVAR (Table 2). The patients in the favorable aortic remodeling group showed a complete thrombosis of the false lumen. The patients in the other group, with adverse aortic remodeling, had an incomplete thrombosis of the false lumen. The RI and the true lumen volume were significantly higher in the favorable group than in the adverse group (1.92 \pm 0.44 vs $0.96 \pm 0.34$, and $224.18 \pm 29.41$ vs $170.91 \pm 37.56 \mathrm{~mL}$, respectively, all $\mathrm{P}<$ $0.001)$, while the false-lumen volume was smaller in the favorable group (120.76 \pm 21.88 vs $188.51 \pm 38.19 \mathrm{~mL}, \mathrm{P}<0.001)$. These results indicate that complete thrombosis of the false lumen promotes favorable aortic remodeling. 
Table 2. Mean true-lumen volume, false-lumen volume, and RI, grouped according to favorable or adverse aortic remodeling in patients with Stanford type B aortic dissection.

\begin{tabular}{lccc}
\hline & Favorable aortic remodeling $(\mathrm{N}=19)$ & Adverse aortic remodeling $(\mathrm{N}=35)$ & $\mathrm{P}$ value \\
\hline True-lumen volume $(\mathrm{mL})$ & $224.18 \pm 29.41$ & $170.91 \pm 37.56$ & $<0.001$ \\
False-lumen volume $(\mathrm{mL})$ & $120.76 \pm 21.88$ & $188.51 \pm 38.19$ & $<0.001$ \\
$\mathrm{RI}$ & $1.92 \pm 0.44$ & $0.96 \pm 0.34$ & $<0.001$ \\
\hline
\end{tabular}

RI: remodeling index.

\section{Factors affecting aortic remodeling after TEVAR}

Table 3 shows the results from the univariate analyses performed to assess the factors affecting aortic remodeling. A branch originating from the false lumen, the presence of multiple tears, hypertension, and age affected aortic remodeling $(P<0.2)$. These variables were analyzed simultaneously using binomial logistic regression, and the results suggested that a branch originating from the false lumen $(\mathrm{OR}=39.854, \mathrm{P}<0.01)$ and the presence of multiple tears $(\mathrm{OR}=$ 27.394, $\mathrm{P}<0.01$ ) were independent risk factors for adverse aortic remodeling (Table 4).

Table 3. Univariate analysis of factors that result in favorable or adverse aortic remodeling in patients with Stanford type B aortic dissection after TEVAR.

\begin{tabular}{|c|c|c|c|}
\hline & Favorable aortic remodeling $(\mathrm{N}=19)$ & Adverse aortic remodeling $(\mathrm{N}=35)$ & $P$ value \\
\hline Gender [male, N (\%)] & $15(78.9)$ & $25(71.4)$ & 0.397 \\
\hline Age $[\mathrm{N}(\%)]$ & $50.74 \pm 13.35$ & $56.34 \pm 11.70$ & 0.116 \\
\hline Branch originating from the false lumen [N (\%)] & $3(15.8)$ & $25(71.4)$ & $<0.001$ \\
\hline Multiple tears $[\mathrm{N}(\%)]$ & $4(26.7)$ & $26(74.3)$ & $<0.001$ \\
\hline Hypertension [N (\%)] & $17(89.5)$ & $22(62.9)$ & 0.035 \\
\hline Smoking $[\mathrm{N}(\%)]$ & $14(73.7)$ & $23(65.7)$ & 0.388 \\
\hline Cerebrovascular disease $[\mathrm{N}(\%)]$ & $5(17.2)$ & $5(14.3)$ & 0.233 \\
\hline Peripheral vascular disease [N (\%)] & $4(21.0)$ & $7(20.0)$ & 0.595 \\
\hline Diabetes mellitus $[\mathrm{N}(\%)]$ & $8(27.6)$ & $10(28.6)$ & 0.239 \\
\hline Renal dysfunction [N (\%)] & $1(5.3)$ & $2(5.6)$ & 0.720 \\
\hline
\end{tabular}

Table 4. Multivariate analysis of factors that result in favorable or adverse aortic remodeling in patients with Stanford type B aortic dissection after TEVAR.

\begin{tabular}{lrrrr}
\hline & OR & & \multicolumn{2}{c}{$95 \% \mathrm{Cl}$} \\
\cline { 3 - 5 } & & Lower limit & Upper limit & P value \\
\hline Age & 1.008 & 0.934 & 1.088 & 0.842 \\
Branch originating from the false lumen & 39.854 & 3.943 & 402.840 & 0.002 \\
Multiple tears & 27.394 & 2.718 & 276.152 & 0.005 \\
Hypertension & 0.318 & 0.037 & 2.724 & 0.296 \\
\hline
\end{tabular}

OR: odds ratio; $95 \% \mathrm{Cl}$ : $95 \%$ confidence interval.

\section{DISCUSSION}

The factors affecting the prognosis of patients with Stanford type B aortic dissection treated using TEVAR remain to be elucidated. The results of this study showed that patients with complete thrombus formation in the false lumen had a more favorable RI. This study suggests that among the examined variables, the presence of a branch emerging from the false lumen and the presence of at least two tear sites are independent risk factors for an adverse RI, characterized 
by an incomplete thrombus formation in the false lumen. The major aims of therapy for aortic dissection include the complete obliteration of the tear, the formation of a thrombus in the false lumen, enlargement of the volume of the true lumen, and reduction in the volume of the false lumen, which are all factors that lead to favorable aortic remodeling (Huptas et al., 2009). The conventional treatment approaches for Stanford type B aortic dissection include open surgery, TEVAR, and conservative medical treatments. TEVAR has recently gained widespread acceptance because of its lower postoperative morbidity and mortality (MacKenzie et al., 2004; Trimarchi et al., 2006; Schoder et al., 2007; Rodriguez et al., 2008). Recent studies have reported that the current proximal closure rate after TEVAR is approximately $77-100 \%$ (Melissano et al., 2008; Rodriguez et al., 2008; Zipfel et al., 2008; Ehrlich et al., 2010; Younes et al., 2010; Tang et al., 2011). Studies have also shown that complete thrombus formation in the false lumen indicated a good prognosis (Erbel et al., 1993; Pretre and Von Segesser, 1997; Dake et al., 1999; Tsai et al., 2007; Zipfel et al., 2008), whereas the lack of thrombus or incomplete thrombus formation in the false lumen was a cause of rupture (Ergin et al., 1994; Tsai et al., 2007). Only 19 patients in this study displayed complete thrombus formation (35\%). The postoperative RI was higher in which set of patients compared to that in patients showing incomplete thrombosis, suggesting better aortic remodeling in patients with complete thrombosis. Indeed, the rapid closure of the proximal tear and the torn intima close to the aortic wall following placement of stent-grafts after TEVAR resulted in the gradual formation of a thrombus in the false lumen. When the thrombus is completely formed in the false lumen, and when the false lumen is completely isolated from the true lumen, the pressure in the false lumen is lower than that in the true lumen, thus favoring aortic remodeling. It is generally believed that the aortic, as well as the true- and false-lumen diameters are difficult to assess accurately after TEVAR. However, Armon et al. (1998) reported that the postoperative changes in true- and false-lumen volumes can be relatively accurately assessed by CTA measurement. Huptas et al. (2009) further proposed that the RI could reflect aortic remodeling, and observed an increase in the RI of patients subjected to TEVAR. However, the RI showed minimal changes in patients undergoing conservative treatments, indicating that TEVAR, but not conservative treatments, promotes aortic remodeling. In this study, the postoperative RI was higher in all patients compared to their preoperative RI, which was consistent with the findings of previous studies (Armon et al., 1998; Huptas et al., 2009). Furthermore, the RI was higher in the complete thrombus formation group than in the incomplete thrombus formation group, suggesting poor aortic remodeling in patients with incomplete thrombus formation. A considerable proportion of patients had more than one tear. Quint et al. (2003) showed that $62 \%$ of their patients had two or more tears. Fifty-seven percent of the patients in this study had multiple tears: when the proximal tear was completely blocked, the distal tear remained in order to allow communication between the false and true lumen, thus impairing the pressure decrease in the false lumen. In this case, incomplete thrombosis of the false lumen hindered aortic remodeling. The results of this study suggest that the preoperative presence of multiple tears was an independent factor affecting aortic remodeling. Very few previous studies have suggested that branch vessels originating from the false lumen might influence aortic remodeling. Miller et al. (1984) reported that the renal artery and the superior mesenteric artery originating from the false lumen were independent risk factors for death in patients with aortic dissection. However, the relationship between branch vessels originating from the false lumen and aortic remodeling remained unclear. Indeed, blood circulation between the visceral artery and the false lumen affects the complete formation of a thrombus, thereby hindering aortic remodeling. Accordingly, the results of this study indicated that branch vessels originating from the false lumen are an independent risk factor affecting aortic remodeling. 
This study is not without limitations. The sample size was relatively small, and the single-center aspect might induce selection and treatment bias. Furthermore, the follow-up period was relatively short. Studies with a larger sample size and a longer follow-up are needed, especially in patients displaying unfavorable aortic remodeling after TEVAR.

In conclusion, Stanford type B aortic dissections that displayed complete thrombus formation after TEVAR showed better and more favorable aortic remodeling. A branch originating from the false lumen and the presence of multiple tears were determined to be independent risk factors for unfavorable aortic remodeling after TEVAR in patients with Stanford type B aortic dissection.

\section{Conflicts of interest}

The authors declare no conflict of interest.

\section{REFERENCES}

Armon MP, Yusuf SW, Whitaker SC, Gregson RH, et al. (1998). Thrombus distribution and changes in aneurysm size following endovascular aortic aneurysm repair. Eur. J. Vasc. Endovasc. Surg. 16: 472-476.

Bickerstaff LK, Pairolero PC, Hollier LH, Melton LJ, et al. (1982). Thoracic aortic aneurysms: a population-based study. Surgery 92: 1103-1108.

Czermak BV, Mallouhi A, Perkmann R, Steingruber IE, et al. (2004). Serial CT volume and thrombus length measurements after endovascular repair of Stanford type B aortic dissection. J. Endovasc. Ther. 11: 1-12.

Daily PO, Trueblood HW, Stinson EB, Wuerflein RD, et al. (1970). Management of acute aortic dissections. Ann. Thorac. Surg. 10: 237-247.

Dake MD, Kato N, Mitchell RS, Semba CP, et al. (1999). Endovascular stent-graft placement for the treatment of acute aortic dissection. N. Engl. J. Med. 340: 1546-1552.

Ehrlich MP, Dumfarth J, Schoder M, Piquet P, et al. (2010). Midterm results after endovascular treatment of acute, complicated type B aortic dissection: the Talent Thoracic Registry. Ann. Thorac. Surg. 90: 1444-1448.

Eid-Lidt G, Gaspar J, Melendez-Ramirez G, Cervantes SJ, et al. (2013). Endovascular treatment of type B dissection in patients with Marfan syndrome: mid-term outcomes and aortic remodeling. Catheter Cardiovasc. Interv. 82: E898-905.

Erbel R, Oelert H, Meyer J, Puth M, et al. (1993). Effect of medical and surgical therapy on aortic dissection evaluated by transesophageal echocardiography. Implications for prognosis and therapy. The European Cooperative Study Group on Echocardiography. Circulation. 87: 1604-1615.

Erbel R, Alfonso F, Boileau C, Dirsch O, et al. (2001). Diagnosis and management of aortic dissection task force on aortic dissection, European Society of Cardiology. Eur. Heart J. 22: 1642-1681.

Ergin MA, Phillips RA, Galla JD, Lansman SL, et al. (1994). Significance of distal false lumen after type A dissection repair. Ann. Thorac. Surg. 57: 820-824; discussion 825.

Fattori R, Tsai TT, Myrmel T, Evangelista A, et al. (2008). Complicated acute type B dissection: is surgery still the best option?: a report from the International Registry of Acute Aortic Dissection. JACC Cardiovasc. Interv. 1: 395-402.

Huptas S, Mehta RH, Kuhl H, Tsagakis K, et al. (2009). Aortic remodeling in type B aortic dissection: effects of endovascular stent-graft repair and medical treatment on true and false lumen volumes. J. Endovasc. Ther. 16: 28-38.

MacKenzie KS, LeGuillan MP, Steinmetz OK and Montreuil B (2004). Management trends and early mortality rates for acute type B aortic dissection: a 10-year single-institution experience. Ann. Vasc. Surg. 18: 158-166.

Melissano G, Bertoglio L, Kahlberg A, Baccellieri D, et al. (2008). Evaluation of a new disease-specific endovascular device for type B aortic dissection. J. Thorac. Cardiovasc. Surg. 136: 1012-1018.

Meszaros I, Morocz J, Szlavi J, Schmidt J, et al. (2000). Epidemiology and clinicopathology of aortic dissection. Chest 117: 1271-1278.

Miller DC, Mitchell RS, Oyer PE, Stinson EB, et al. (1984). Independent determinants of operative mortality for patients with aortic dissections. Circulation 70: 1153-164.

Miller LE and Pierson LM (2011). Discordance of aortic remodeling with clinical outcomes in patients treated with endovascular repair for uncomplicated type B aortic dissection. Interact. Cardiovasc. Thorac. Surg. 13: 203-204.

Nienaber CA and Eagle KA (2003). Aortic dissection: new frontiers in diagnosis and management: Part I: from etiology to diagnostic strategies. Circulation 108: 628-635.

Pretre R and Von Segesser LK (1997). Aortic dissection. Lancet 349: 1461-1464. 
Quint LE, Platt JF, Sonnad SS, Deeb GM, et al. (2003). Aortic intimal tears: detection with spiral computed tomography. J. Endovasc. Ther. 10: 505-510.

Rodriguez JA, Olsen DM, Lucas L, Wheatley G, et al. (2008). Aortic remodeling after endografting of thoracoabdominal aortic dissection. J. Vasc. Surg. 47: 1188-1194.

Schoder M, Czerny M, Cejna M, Rand T, et al. (2007). Endovascular repair of acute type B aortic dissection: long-term followup of true and false lumen diameter changes. Ann. Thorac. Surg. 83: 1059-1066.

Singh-Ranger R, McArthur T, Corte MD, Lees W, et al. (2000). The abdominal aortic aneurysm sac after endoluminal exclusion: a medium-term morphologic follow-up based on volumetric technology. J. Vasc. Surg. 31: 490-500.

Tang JD, Huang JF, Zuo KQ, Hang WZ, et al. (2011). Emergency endovascular repair of complicated Stanford type B aortic dissections within 24 hours of symptom onset in 30 cases. J. Thorac. Cardiovasc. Surg. 141: 926-931.

Trimarchi S, Nienaber CA, Rampoldi V, Myrmel T, et al. (2006). Role and results of surgery in acute type B aortic dissection: insights from the International Registry of Acute Aortic Dissection (IRAD). Circulation 114: I357-364.

Tsai TT, Evangelista A, Nienaber CA, Myrmel T, et al. (2007). Partial thrombosis of the false lumen in patients with acute type B aortic dissection. N. Engl. J. Med. 357: 349-359.

Wheatley JM, Rosenfield NS, Heller G, Feldstein D, et al. (1995). Validation of a technique of computer-aided tumor volume determination. J. Surg. Res. 59: 621-626.

Yang CP, Hsu CP, Chen WY, Chen IM, et al. (2012). Aortic remodeling after endovascular repair with stainless steel-based stent graft in acute and chronic type B aortic dissection. J. Vasc. Surg. 55: 1600-1610.

Younes HK, Harris PW, Bismuth J, Charlton-Ouw K, et al. (2010). Thoracic endovascular aortic repair for type B aortic dissection. Ann. Vasc. Surg. 24: 39-43.

Zipfel B, Buz S, Hammerschmidt R, Krabatsch T, et al. (2008). Early clinical experience with the E-vita thoracic stent-graft system: a single center study. J. Cardiovasc. Surg. 49: 417-428. 